\title{
The Building Blocks of Architecture and Mathematics
}

\author{
Kim Williams ${ }^{1}$
}

Published online: 22 July 2021

(c) Kim Williams Books, Turin 2021

\begin{abstract}
Editor-in-Chief Kim Williams examines the sometimes very sophisticated use of fundamental mathematical elements-curves, grids, simple polygons and polyhedra-in ancient and contemporary architecture and introduces the articles in Nexus Network Journal vol. 23 no. 3 (2021).
\end{abstract}

It is sometimes surprising that fundamental mathematical elements such as curves, grids, simple polygons and polyhedra can perform so many different functions in the world of architecture. The papers in this issue of the Nexus Network Journal feature sophisticated uses of these elements in architecture and urban planning, both ancient and modern.

In "Surface Discretisation with Rectifying Strips on Geodesics", Andrés MartínPastor and Francisco González-Quintial present a graphic model to discretize surfaces using developable straight strips to cover a surface tangentially, optimizing material consumption and offering a wide range of applications. Their research is based on the concept of a "rectifying surface", a special developable surface that rests on any curve.

Maja Petrović, Branko Malešević and Radovan Štulić, in "Weberian FocalDirectorial Curves: Geometric Genesis and Form Variation", present a discussion of new plane curves (multi-directorial curves as counterpart to multi-focal curves and focal-directorial curves as curves of a transitory type) through their geometric genesis and their form variation. The paper illustrates how the properties of the curves allow them to be used as patterns for designing more complex forms as well as for plane tiling.

In 1968 Andre Barre and Albert Flocon depicted a square room from its interior by means of a $360^{\circ}$ total perspective. In "The $360^{\circ}$ Curvilinear Perspective: A Hybrid Hypercubic Angular Space Grid Based on the 1968 Barre and Flocon proposal" Julián Santoyo and Miguel A. Santoyo return to that proposal to reveal how this specific case of a pseudo-spherical projection for a hypercube was achieved by a

Kim Williams

kwb@kimwilliamsbooks.com

1 Kim Williams Books, Corso Regina Margherita, 72, 10153 Turin, Italy

Birkhäuser 
hybrid using ellipse curves in one of the spatial directions and a different spherical projection in the other direction. Their results pave the way to possible new interpretations of the built environment.

In this issue's Geometer's Angle column, Tuğrul Yazar presents “Compass Construction of Bézier Curves and B-Splines", in which constructions aim to translate the B-Spline definition of Paul de Casteljau into the modified versions of compass-only constructions explained by Aleksandr Kostovskii. Yazar sees his research as providing a basic stepping stone for a broader and unifying understanding of distinct architectural geometries.

Patterns of simple elements into more complex arrangements can create systems that are both strong and flexible.

Francesca Lecci, Cecilia Mazzoli, Cristiana Bartolomei and Riccardo Gulli, in "Design of Flat Vaults with Topological Interlocking Solids" investigate the principles that regulate complex stereotomic constructions as a starting point for the design of a new two-dimensional floor structure based on the principles of topological interlocking materials, that is, based on structures that use polyhedra as repeated modules to form structurally stable planes.

Dario Parigi presents "The Parigi Mechanism: Properties and Application of a Kinetic Reciprocal System (KRS) Adaptive Façade", a novel mechanism which he invented, possessing one degree of freedom and described by means of the kinetic reciprocal system (KRS) algorithm. The mechanism consists of an infinitely extendable hexagonal network of elements connected with pin-slot joints, and lends itself well to kinetic architecture, that is, buildings or building components that have variable location or mobility and/or variable geometry or movement.

In addition to their use as building forms, simple elements, such as lines forming axes or grids, can also underline ideological and symbolic intents.

Such symbolic notions were certainly exploited by ancient architects, as Norma Camilla Baratta and Giulio Magli show in "The Role of Astronomy and Feng Shui in the Planning of Ming Beijing". Thanks to the technologies of satellite imagery and palaeomagnetic data analysis, they were able to determine the relationship of symbolic aspects of the planning of the city, astronomical alignments and the doctrine of feng shui. Their research shows that orientations of the axes of the "cosmic" temples and of the Forbidden City were most likely magnetic, while astronomy was used in topographical connections between the temples and in the plan of the Forbidden City itself.

Rizal Muslimin, in "Cultural Computation on the Traveling Hamlet: Ciptarasa and Ciptagelar", describes a development pattern analysis of two traditional villages, one of which was the predecessor of the other. He used computer modelling to explain how the formal and informal cultural elements intermingle and shape the settlement pattern. Interpreting rules from the earlier capital and using them to identify a pattern in the current capital allows him to synthesize the rules and demonstrate rule iteration across scales, indicating a dynamic relationship between the settlement and its cosmology.

Such cosmic connections seem to be lost in architecture today, but there are still those who search for a way to continue its relevance. This theme is developed in "Cosmogenic Pattern Language: Toward an Architectural Language Based on the 
Cosmogenic Patterns of Pre-Modernism" by Diana Allam, Yasmine S. Hegazi, Mohamed Atef Abo-Ashour and Mahmoud Fathi Elalfi, who present the framework for a contemporary architectural language that uses the notion of cosmogenic architecture. This notion suggests that architecture might be related to key elements of the sky and designed according to geometric patterns captured from the forms that abstract the cosmic order.

José Vela Castillo and Pedro García Martínez discovered that military bases built during the Cold War by the United States at various points around the globe share a common design project which implements an underlying grid-like system in the organization of their masterplans. In "The Use of Orthogonal Grids in the Design of US Military Bases in Spain" they argue that this grid-like system was conceived as a sophisticated and precise graphic tool able to facilitate the design, construction, and maintenance of the bases, but also to further an ideological project.

Finally, in all applications of mathematical and cosmological notions to architecture there is an underlying push-pull between order and complexity: too much order can make our built environment boring and unchallenging; too much complexity can lead to a sense of chaos and lack of meaning. Kahina Meddahi and Kenza Boussora address this in "Aesthetic Measures Of Algiers' Colonial Facades", in which Birkhoff's aesthetic measure based on order and complexity was applied to assess a group of facades. The aim is to ultimately be able to elaborate a guideline of design principles for the preservation of both buildings and their specific context.

As this issue is being prepared, preparations are also underway for the Nexus $20 / 21$ conference on architecture and mathematics, which will take place in an online format from 26 to 29 July 2021. The name "Nexus 20/21" reflects the fact that the 2020 conference was postponed to 2021 because of the COVID-19 pandemic. Future issues of the $N N J$ will bring readers the very best of the many presentations at the conference.

Publisher's Note Springer Nature remains neutral with regard to jurisdictional claims in published maps and institutional affiliations.

Kim Williams received her degree in Architectural Studies from the University of Texas in Austin. She became interested in mathematics and architecture while writing Italian Pavements: Patterns in Space (Anchorage Press, 1997) about the role of decorated pavements in the history of Italian architecture, and it has been her field of research ever since. She is the founder and director of the international, interdisciplinary conference series "Nexus: Relationships Between Architecture and Mathematics", and is the founder and co-editor-in-chief (with Michael Ostwald) of the Nexus Network Journal. She is the author of Daniele Barbaro's Vitruvius of 1567 (Birkhäuser, 2019). Her forthcoming book, with Cosimo Montelone, is Daniele Barbaro's Perspective of 1568 (Birkhäuser, 2021). 